\title{
A Multifractal Inverse Problem Applied to Heart Rate Data Synthesis
}

\author{
DC Lin ${ }^{\dagger}, \mathrm{J}$ Thevaril ${ }^{*}$ \\ ${ }^{\dagger}$ Department of Mechanical, Aerospace \& Industrial Engineering, ${ }^{\dagger}$ Department of \\ Electrical Engineering, Ryerson University, Toronto, Canada
}

\begin{abstract}
Daytime heart rate variability synthesis is proposed based on the assumption of multiplicative cascade. The parameters of the cascade were extracted from healthy $R R$ intervals (RRi) to generate the synthetic data which reproduce some of the known HRV phenomenology, such as the multifractal scale invariance, stretch exponentialexponential-gaussian increment distribution transition from small to large increments, and Poisson excursion in small RRi fluctuation. However, this model only tackles the broad-band l/f-like fractal component of HRV. Narrow-band components such as the respiration were not considered.
\end{abstract}

\section{Introduction}

The random fluctuation of the interbeat time (RRi) interval reveals the complex cardiovascular dynamics in humans. RRi has been widely studied by its power spectrum, which is known to consist of a narrow-band harmonic component and a broad-band fractal component [1]. In long-term HRV, the fractal component accounts for a large percentage of the total RRi signal power. Although its origin and physiological function of remain largely unclear, experimental data suggest that the HRV fractal component represents a vital factor as the deficit of the fractal characteristic implies a higher mortality rate in a number of heart disease conditions [2].

The proposed model is motivated by the finding of multifractal in healthy daytime RRi records $[3,4]$. The potential of using multiplicative cascade to model the generating mechanism of HRV was thus raised [4]. For the CINC 2002 challenge, a random bounded cascade on the dyadic scale is developed to model (only) the fractal component in long-term HRV. The synthesis implies a multifractal inverse problem to define the cascade based on the experimental RRi data. In this study, we used the log-increment of the RRi to achieve the parameter identification for the cascade. The synthetic RRi so produced is able to exhibit some of the crucial statistics of healthy daytime HRV.

This paper is organised in five sections. The bounded cascade model and parameter estimation are explained in Sec. 2 and 3 respectively. In Sec 4, the experimental and simulation results are given. Concluding remarks are given in Sec 5.

\section{Bounded cascade}

Random cascade can be generally put in the framework of positive martingale theory [5]. In construction, it consists of three basic elements: the multiplicative data generation rule, the probability law of the cascade component and the branching rule. Let $r_{J}(t)$ denote the synthetic RRi between the $t^{\text {th }}$ and $(t+1)^{\text {th }}$ beat. The cascade HRV assumes the random phenomenon is a result of the product of $J$ cascade components:

$$
r_{J}(t)=\prod_{j=1}^{J} \omega_{j}(t)
$$

The cascade component can be written as $\omega_{j}(t)=1+\xi_{j}$ where $\xi_{j}, j=1, \ldots, \mathrm{J}$ are independent gaussian variables (in $j$ ) with $\left\langle\xi_{j}>=0,\left\langle\xi_{i} \xi_{j}>=\delta_{i j} \sigma_{j}^{2}\right.\right.$ ( $\delta_{i j}$ is the Kronecker delta). Our experience with other distributions does not indicate qualitatively different results. A bounded $r_{J}(t)$ is imposed based on the refractory property of the heart muscle cells [6]. For finite cascade $J<\infty$, this is achieved since $\left\{\sup \left|\omega_{j}(t)\right|<\infty\right\}$ holds almost surely. However, we like to assume a stronger condition to assure boundedness even in the $J \rightarrow \infty$ limit. Motivated by the deterministic bounded cascade [7], a power law decay in the component variance is imposed: $\sigma_{j}=\sigma_{0} 2^{-\alpha(j-1)}$ where $\sigma_{0}$ and $\alpha$ are constants to be determined. Intuitively, the decreasing $\sigma_{j}$ creates a damping effect by the small time scale components to prevent $r_{J}(t)$ from reaching unbounded values in the $J \rightarrow \infty$ limit. As a result, a large amplitude jump in the small time scale component becomes less likely, making the large time scale component the leading factor for large scale fluctuation in $r_{J}(t)$. The range of healthy and diseased HRV applies to 
the parameter space $\sigma_{j}<1$. For $\sigma_{j}>1$, the simulated data is too "intermittent" to be considered for HRV.

In addition to the gaussian assumption and the decaying variance, $\omega_{j}(t)$ is further set to vary only on discrete times $\left\{t_{k}^{(j)}\right\}: \omega_{j}(t)=\omega_{j}\left(t_{k}^{(j)}\right)$ for $t_{k}^{(j)} \leq t<t_{k+1}^{(j)}$. The time set $\left\{t_{k}^{(j)}\right\}$ is designed to provide the self-similarity in the data fluctuation and thus defines the branching rule for the cascade. In this work, dyadic time scales were used: $t_{k}^{(j)}=$ $k N / 2^{j}, k=1, \ldots, 2^{j}$ where $N=2^{J}$ is the number of data points. Notice that the number of the elements of $\left\{t_{k}^{(j)}\right\}$ is an increasing function of $j$. Hence, the $\omega_{j}(t)$ for small $j$ can be referred to as the large time scale component and is said to give rise the "slow dynamics." Similarly, the $\omega_{j}(t)$ of large $j$ is referred to as the small time scale component which gives rise to the "fast dynamics." In this view, it is tempting to relate the "slow" and "fast" dynamics of the cascade to the sympathetic and parasympathetic nervous system activities. Such a connection was recently tested and consistent results with the experimental observations reported in the past were found $[4,8]$.

\section{Parameter estimation}

$\alpha$ and $\sigma_{0}$ in $\sigma_{j}$ are the two model parameters that need to be extracted from the experimental RRi to simulate the fractal component of HRV. In what follows, we will present a procedure to estimate these parameters. After they are determined, we then check the scaling of the increment, $\left\langle\left|\Delta r_{J}(\tau)\right|^{q}\right\rangle \sim \tau^{\zeta(q)}$ where $\Delta r_{J}(\tau)=r_{J}(t+\tau)-$ $r_{J}(t)$, to verify the generation of multifractal and other HRV phenomenology. For $\alpha$, it is estimated from the time averaged statistics of the log-increment of $\log _{2}\left(r_{J}(t)\right)$. Let $y_{J}(\tau ; t)=\log _{2}\left(r_{J}(t+\tau)\right)-\log _{2}\left(r_{J}(t)\right)$ for integers $\tau$, $t$. The time averaged $p$ th order moment of $y_{J} \quad(p>0)$ can be written as

$$
m_{\tau}^{(p)} \sim \frac{1}{N} \sum_{t} y_{J}(\tau, t)^{p}=\frac{1}{N} \sum_{t}\left[\sum_{j} \rho_{j}(\tau, t)\right]^{p}
$$

where

$$
\rho_{j}(\tau, t)=\log _{2}\left(\frac{\omega_{j}(t+\tau)}{\omega_{j}(t)}\right) .
$$

For $\tau_{k}=N / 2^{k}$, it can be shown that the set $T_{k}^{(j)}=\left\{t ; \omega_{j}\left(t+\tau_{k}\right)\right.$ $\left.\neq \omega_{j}(t)\right\}$ consists of disjoint segments, each of which associates with an unique $\rho_{j}\left(\tau_{k}, t\right)$ value. Moreover, $\left|T_{k}^{(j)}\right|$ $=\left(2^{j}-1\right) \times \tau_{k}$ for $j \leq k$ and $\left(2^{j}-2^{j-k}\right) \tau_{k}$ for $j>k$. The contribution from the $j^{\text {th }}$ cascade component to the second moment can be given by

$$
\frac{1}{N} \sum_{t=1}^{N}\left[\rho_{j}\left(\tau_{k} ; t\right)\right]^{2}=\frac{1}{N} \sum_{t \in T_{k}^{(j)}}^{N}\left[\rho_{j}\left(\tau_{k} ; t\right)\right]^{2}
$$

$$
\sim \frac{\left|T_{k}^{(j)}\right|}{N}\left\langle\left[\log _{2}\left(\frac{1+\xi_{j}^{\prime}}{1+\xi_{j}}\right)\right]^{2}\right\rangle
$$

Substituting (4) into (2) and keeping only second order terms result in

$$
\begin{aligned}
& m_{\tau_{k}}^{(2)} \sim 2 \sum_{j} \frac{\left|T_{k}^{(j)}\right|}{N} \sigma_{j}^{2} \\
& \sim \frac{\left|T_{k}^{(j)}\right|}{N}\left[\frac{2\left(1-2^{u k}\right)}{D_{1}}-\frac{1-2^{\alpha k}}{D_{2}}+\left(2^{k}-1\right) \frac{\alpha k-2^{\alpha J}}{D_{2}}\right]
\end{aligned}
$$

where $u=1-2 \alpha, D_{1}=1-2^{u}$ and $D_{2}=1-2^{-2 \alpha}$. For $\alpha \ll 1$, $D_{2} \ll D_{1}$, the third term is the dominant term in (5) due to the factor $2^{k}-1$. For large $J » 1$, it can be shown that $\sigma_{y}\left(\tau_{k}\right) \sim \tau_{k}^{\alpha}$ holds. Once $\alpha$ is estimated, $J$ is calculated based on $N$ and $\sigma_{0}$ is determined accordingly.

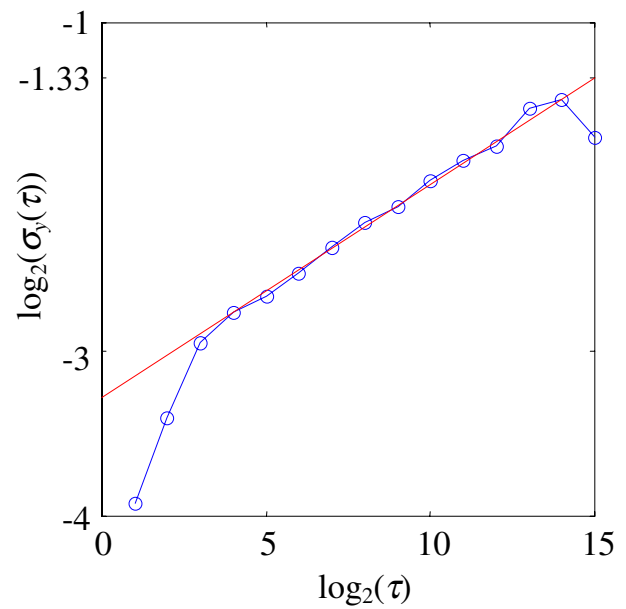

Figure $1 \sigma_{y_{k}}\left(\tau_{k}\right) \sim \tau_{k}^{\alpha}$ from a data set in DB1.

\section{HRV synthesis}

From the last section, we estimated the cascade parameters from two different RRi databases: DB1 which consists of the day-time ambulatory ECG recording from ten healthy young adults conducting normal daily activities, and DB2 which was downloaded from the normal sinus rhythm database in physionet. Fig. 1 shows the power law $m_{\tau_{k}}^{(2)}=\log _{2}\left(\sigma_{y}\left(\tau_{k}\right)\right) \sim \tau_{k}^{\alpha}$ from a typical data set in DB1. The exponent $\alpha$ is estimated from the $\log -\log$ plot of $\sigma_{y}\left(\tau_{k}\right)$ versus $\tau_{k}$ and $\sigma_{0}$ is estimated from the intercept at the largest time scale. In the submission to CINC 2002 competition, we used the group-averaged $\sigma_{0}$ $=0.329$ and $\alpha=0.126$ to generate the synthetic RRi. Fig. 
2 shows the typical $r_{J}(t)$ generated by the multiplicative cascade. This time series is able to capture similar HRV phenomenology of the experimental data [4]: (a) stretch exponential-exponential-gaussian (SEG) transition in $\Delta r_{J}(\tau)$ distribution as $\tau$ increases (Fig. 3), (b) multifractal scaling in $\left\langle\left|\Delta r_{J}(\tau)\right|^{q}\right\rangle \sim \tau^{\zeta(q)}$ with nonlinear $\zeta(q)$ (Figs. 4a, $4 b)$ and (c) Poisson excursion of small RRi increment (not shown).

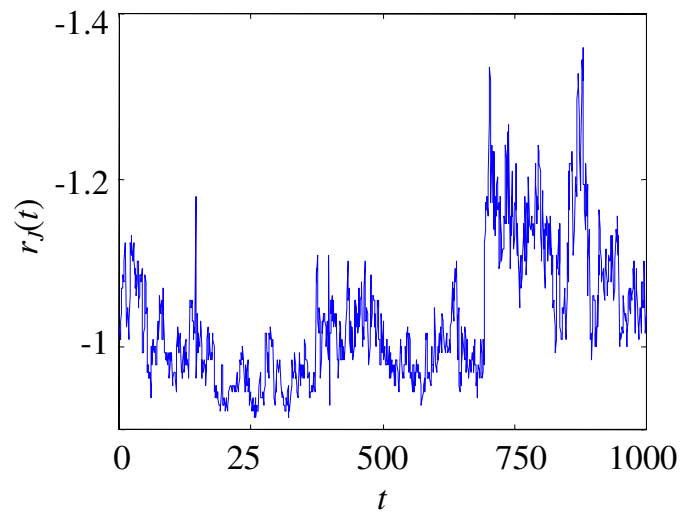

Figure 2 Artificial RRi based on multiplicative cascade. The cascade parameters are $\log _{2}\left(\sigma_{0}\right)=-1.7, \alpha=0.1264$.

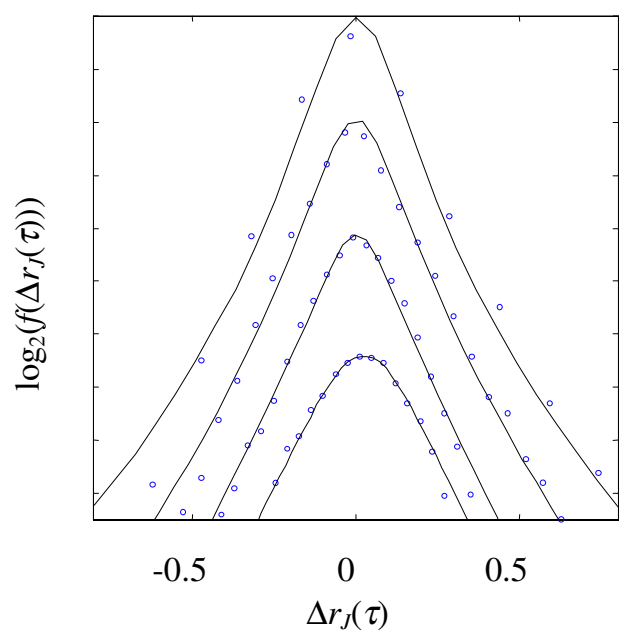

Figure 3 SEG transition of the increment probability density function (PDF) for $\tau=1,8,128,2048$ (from top to bottom). The "o" are the estimated PDF from the data used in Fig. 2. Solid lines are the estimated PDF of cascade $\Delta r_{J}(\tau)$ using the same parameters as Fig. 2. Each increment PDF $f\left(\Delta r_{J}(\tau)\right)$ are rescaled and moved vertically for clarity purposes.

\section{Conclusion}

In this study, RRi synthesis was conducted based on the assumption of cascade HRV. The artificial data successfully captured some of the crucial properties in day-time healthy RRi fluctuation. The assumptions made in the current model include: (a) normally distributed cascade components, (b) power law decay in the component variance and (c) dyadic branching rule. These properties can be perturbed and give qualitatively similar results [8]. It should be noted that the model aims only at the fractal component of HRV. The so-called harmonic components were not considered. As a result, the power spectrum of $r_{J}(t)$ exhibits theoretically a perfect $1 / \mathrm{f}$ law.
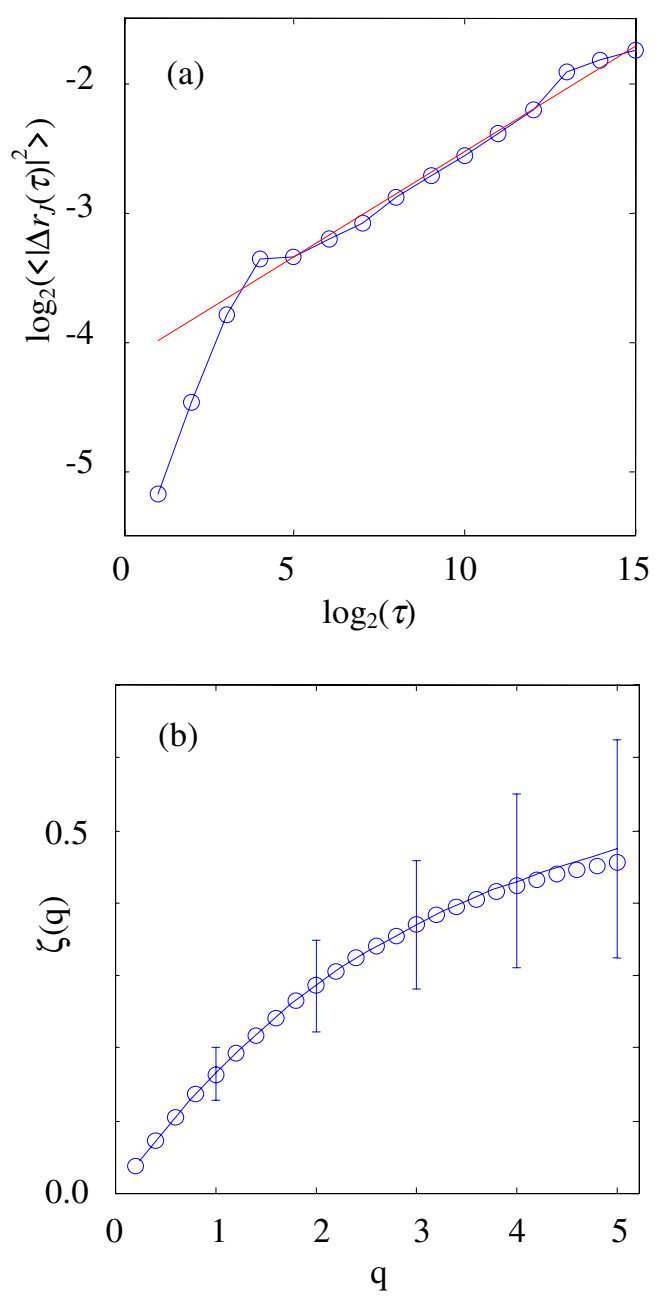

Figure 4 (a) Power law scaling of $\left\langle\left|\Delta r_{J}(\tau)\right|^{q}\right\rangle$ in a typical data set of DB1. (b) Averaged $\zeta(q)$ from 100 samples of $r_{J}(t)$ generated by the cascade whose parameters are determined by the method described in Section 3 (showing as solid line and one standard deviation). $\zeta(\mathrm{q})$ estimated from the real RRi data is plotted in "o". 


\section{Acknowledgements}

This research is supported by the Natural Science and Engineering Research Council of Canada. DCL would like to thank Dr. A.L. Goldberger for bringing the attention of the challenge and Dr. G. Moody for valuable suggestions on meeting the rules of event I competition.

\section{References}

[1] Yamamoto Y, Hughson RL. Extracting fractal component from time series. Physica D 1993;68:250-264.

[2] Task Force of the ESC and NASPE. Standards and measurements of heart rate variability. Euro. Heart J. 1996;17:354-379.

[3] Ivanov PCh, Amaral LAN, Goldberger AL, Havlin S, Rosenblum MG, Struzik SR, Stanley HE. Multifractality in human heartbeat dynamics. Lett. to Nature 1999;399:461-465.

[4] Lin DC, Hughson RL. Modeling heart rate variability in healthy humans, a turbulence analogy. Phys. Rev. Lett. 86; 2001:1650-1653; Lin DC, Hughson RL. A phenomenology model for normal sinus rhythm in healthy humans. IEEE Tran. Biomed. Engng. 2002;49:97-114.

[5] Waymire EC, Williams SC. A general decomposition theory for random cascades. Bull. Am. Math. Soc. 1994;31:216-222.

[6] Little RC and Little WC. Physiology of the heart and circulation. Year Book Med. Publ. Inc.;1989.

[7] Marshak A, Davis A, Cahalan R, Wiscombe W. Bounded cascade models as nonstationary multifractals. Phys. Rev. E 1994;49:55-81.

[8] D.C. Lin. Robustness and perturbation in cascade heart rate variability. submitted for publication.

Address for correspondence.

D.C. Lin

Department of Mechanical, Aerospace \& Industrial Engineering Ryerson University

350 Victoria St.

Toronto, Ontario, Canada

derlin@acs.ryerson.ca 